\title{
Evaluation of the Performance of Two Selective Enrichment Media and Two Selective Plating Media for the Detection of Salmonella from Primary Poultry Production, According to ISO 6579:2002
}

\author{
Nchawa Yangkam Yhiler¹, Enya Bassey Bassey1,2*, Monday Francis Useh'1 \\ ${ }^{1}$ Department of Medical Laboratory Science, Faculty of Allied Medical Science, University of Calabar, Calabar, \\ Nigeria \\ ${ }^{2}$ World Health Organization, Abuja, Nigeria \\ Email: yankgam@yahoo.com, ${ }^{*}$ bassey69@yahoo.com, francisuse@yahoo.co.uk
}

Received 2 August 2015; accepted 14 September 2015; published 17 September 2015

Copyright (C) 2015 by authors and Scientific Research Publishing Inc.

This work is licensed under the Creative Commons Attribution International License (CC BY).

http://creativecommons.org/licenses/by/4.0/

(c) (1) Open Access

\section{Abstract}

Salmonellosis remains a major problematic food born zoonosis worldwide. Poultry and poultry products have been frequently incriminated as the major sources for the aetiological agent, Salmonella species. The implementation of appropriate surveillance and monitoring programme for food safety ultimately requires the examination of food for the presence of food borne pathogens. This study was aimed at evaluating the effectiveness of Modified Semisolid Rappaport-Vassiliadis (MSRV) and Muller-Kauffmann tetrathionate-novobiocin (MKTTn) selective enrichment media for the isolation of Salmonella, as well as assessment of the performance of xylose-lysine-deoxycholate (XLD) agar as the classical and preferential selective plating medium by comparing it with Brilliant Green (BG) agar for the detection of Salmonella in the primary production of poultry based on the ISO 6579:2002. Three hundred and seventy-four (374) samples from the primary poultry production system underwent the traditional culture method for the detection of Salmonella involving pre-enrichment, selective enrichment, selective plating and confirmation. The rate of recovery of Salmonella from the entire samples was $59.1 \%$. The rate of recovery of Salmonella from XLD and BG selective plating media when MSRV selective enrichment media was used was $\mathbf{9 8 . 2} \%$ and $95.5 \%$ respectively whereas $90.0 \%$ and $64.3 \%$ respectively when MKTTn selective enrichment media was used. The highest sensitivity was observed in the combination of MSRV/XLD (0.98), followed by MSRV/BG (0.95). The combination of MSRV/BG had the highest specificity (0.88) followed by MKTTn/BG (0.65). Based on the results of this study, selective enrichment media play a substantial role on the sensitivity and specificity of the selective plating media. MSRV

*Corresponding author.

How to cite this paper: Yhiler, N.Y., Bassey, E.B. and Useh, M.F. (2015) Evaluation of the Performance of Two Selective Enrichment Media and Two Selective Plating Media for the Detection of Salmonella from Primary Poultry Production, According to ISO 6579:2002. Open Journal of Medical Microbiology, 5, 128-135. http://dx.doi.org/10.4236/ojmm.2015.53016 
was revealed as the most important selective enrichment medium, necessary for the investigation of Salmonella and that its combination with BG selective plating media yielded the best result for the selective isolation of Salmonella in the primary production of poultry.

\author{
Keywords
}

Salmonella, Selective-Enrichment-Media, Selective-Plating-Media

\title{
1. Introduction
}

The poultry production settings have been frequently implicated as major reservoirs for Salmonella species, and Salmonellas still remains a major problematic zoonosis resulting in significant morbidity in both humans and animals worldwide [1] [2]. Zoonotic Salmonellas are the most frequently identified agents causing gastroenteritis in humans and animals worldwide and are responsible for the significant morbidity and mortality in humans and animals resulting in an estimated 1.3 billion cases with approximately 3 million deaths [2] [3]. Human salmonellosis cases have been frequently traced to be due to the consumption of contaminated foods of animal origin, especially poultry and poultry products [4]-[6]. In order therefore to prevent and ultimately eliminate food borne salmonellosis, it would be of great importance to put in place surveillance and monitoring programs for food safety.

Food safety is mainly geared towards the detection of pathogenic microorganisms that may render the food unsuitable for human consumption and the conventional methods for the detection of Salmonella and other food borne pathogens is by the traditional culture methods. These methods have the merits of reliability of the media, easy to perform and the capacity to actually recover the causative agents even at very small numbers [6] [7]. The increasing application of external quality assurance programmes has led to wide use of international standard methods, such as International Organization for Standardization (ISO) 6579:2002 intended for foodstuffs and animal feed stuffs. In recent years, the ISO 6579:2002 standard method for detection of Salmonella from primary animal production has been evaluated and widely adopted by several standard laboratories worldwide [8]. This standard method requires four relevant stages which involves 1) non-selective pre-enrichment in modified Buffered Peptone water (BPW); 2) the selective enrichment in Modified Semisolid Rappaport-Vassiliadis (MSRV) or Muller-Kauffmann tetrathionate-novobiocin broth (MKTTn); 3) the selective plating on xylose-lysine-deoxycholate (XLD) agar and an additional suitable selective plate of choice and; 4) confirmation characteristics [5] [9]-[12].

Previously, the Rappaport-Vassiliadis (RV) enrichment broth was modified to the Rappaport-Vassiliadis Soy (RVS) broth which has currently been further modified into the Modified Semisolid Rappaport-Vassiliadis (MSRV). These amendments were aimed at heightening the reliability of the enrichment medium [12] [13]. The MKTT enrichment broth was the first to develop by Muller andwas later supplemented with Ox bile and Brilliant Green in order to enhance selectivity by Kauffmann and Jeffries further included the addition of novobiocin at $40 \mathrm{mg} / \mathrm{L}$ in order to inhibit indigenous flora, such as Proteus spp., and henceimproving on the rate of recovery of Salmonella [14]-[16]. Two selective solid media are required for the isolation of Salmonella following selective enrichment. The first preferred medium is xylose lysine deoxycholate agar (XLD) and the second can be any other equivalent selective medium capable of recovering lactose-positive Salmonella, S. Typhi, and $S$. Paratyphi strains. These may include Hektoen enteric (HE) agar, bismuth sulfite (BS) agar, and brilliant green (BG) agar etc. [17] [18].

The detection of Salmonella using traditional selective plating media is based on lactose fermentation and hydrogen sulfide $\left(\mathrm{H}_{2} \mathrm{~S}\right)$ production by the organism. However, most media have poor specificity, creating an abundance of false positives and resulting in time-consuming identification [19]. Therefore, to reduce the workload for unnecessary examination of presumptive colonies, and to enable the fast and easy detection of Salmonella, it is of important necessity to investigate which selective plating media will provide results that are closest to the true situation. There are a good number of selective plating media that have been developed on the basis of the biochemical characteristics of Salmonella, such as $\alpha$-galactosidase activity in the absence of $\beta$-galactosidase activity, C8-esterase activity, catabolism of glucuronate, glycerol, and propylene glycol, andhydrolysis of X-5-gal [19] [20]. In spite of the numerous studies that have aimed at determining the optimal selective media 
showing high level of sensitivity and specificity for the detection of Salmonella in food and environmental samples [12], this current study was aimed at evaluating the sensitivity and specificity of Brilliant Green (BG) selective plating medium compared with the preferential selective plating medium, Xylose lysine deoxicholate (XLD). This study determined the rate of recovery of Salmonella from the primary production of poultry and compared the sensitivity, specificity, and the rates of false positives and false negatives of the selective enrichment media (MSRV and MKTTn) and the selective plating media (XLD and BG) for the detection of Salmonella according to ISO 6579:2002.

\section{Materials and Methods}

\subsection{Sample Collection}

From August 2013 and May 2014, three hundred and seventy four (374) samples were randomly collected from three different sources in the poultry production settings within Calabar, Nigeria: out of which 170 were obtained from the poultry environmental sources (poultry feeds, poultry drinking water, poultry litter, poultry abattoir reins and dust from poultry house), 136 were obtained from poultry birds themselves (guts, cloacal swabs, poultry meat and eggs) and 68 were obtained from the poultry personnel (poultry personnel stool and hand washings). The samples were stored in an ice chest container and transported to the laboratory for immediate processing. Samples were collected based on the method as described by Akond et al. [21].

\subsection{Isolation of Salmonella Species}

The isolation of Salmonella species from the poultry production setting in Calabar was based on the ISO 6579:2002 involving the non-selective pre-enrichment stage using 10\% buffered Peptone Water (BPW), the selective enrichment stage using Modified Semisolid Rapapport-Vassiliadis (MSRV) and Muller-Kauffmann Tetrathionate-nivobiocin (MKTTn) selective enrichment media, and finally the selective plating stage using Xylulose Lysine Deoxycholate (XLD) agar and Brilliant Green (BG) selective plating media.

A dilution of 1 in 10 of the samples were prepared in modified buffered peptone water (BPW) and incubated over night at $37^{\circ} \mathrm{C} .1 \mathrm{ml}$ and $0.1 \mathrm{ml}$ of the non-selective enrichment (modified BPW) was inoculated into $9 \mathrm{ml}$ of MKTTn and MSRV selective enrichment media respectively. These were respectively incubated at $37^{\circ} \mathrm{C}$ and $42^{\circ} \mathrm{C}$ in separate incubators for $24 \mathrm{~h}$. After incubation, a loop full from each of the selective enrichment media was streaked onto both Xylose Lysine Desoxycholate (XLD) and Brilliant Green (BG) selective plates.

\subsection{Confirmation of Salmonella Isolates}

Presumptive Salmonella positive colonies on selective plates were picked by means of a sterile wire loop and used to stab and streak on pre-prepared Triple Sugar Iron Agar (TSIA) and Christensen agar (CA) slants. Colonies that produced alkaline slope/acid butt with or with out the production of $\mathrm{H}_{2} \mathrm{~S}$ and gas, on TSIA slant and Urease negative on Christensen agar slant were considered suggestive of Salmonella. Further confirmation was based on the standard biochemical techniques involving Lycine Decarboxilation (LCD) test, $\beta$-alactosidase test, Acetone production test and Indole production test. Finally confirmation was supplemented by serological confirmation involving commercially available polyvalent Salmonella antisera kit (Denka Seiken Co. Ltd. Tokyo, Japan) specific for all group and type-factor Salmonella antigens.

\subsection{Statistical Analysis}

The data generated in this study were analysed by means of the Statistical Package for Social Sciences (SPSS) 20.0 (IBM, USA). The prevalence of Salmonella from the primary production of poultry from the three sources as obtained by the two selective plating media (XLD and BG) were analysed by means of the Fisher's exact test. P-values of less than 0.05 ( $\mathrm{P}<0.05)$ was considered statistically significant.

Selective plating media exhibiting Salmonella colonies was considered presumptive Salmonella positive plates prior to confirmation. Presumptive Salmonella positive plates that showed positive Salmonella colonies following confirmation were considered as true positives and the others as false positive. Among the selective plating media that did not reveal Salmonella colonies, those that demonstrated Salmonella colonies following confirmation were considered false negative and the others true negatives. The Sensitivity (Sc), Specificity (Sp), positive predictive value (PPV) and negative predictive value (NPV) were also analysed according to the study 
carried out by Carrique-Mas et al. [22]. The Sensitivity (Sc) was given as the proportion $(0 \leq \mathrm{Sc} \leq 1)$ of the confirmed Salmonella positive plates that came from the presumptive Salmonella positive plates, while the Specificity (Sp) was given as the proportion $(0 \leq \mathrm{Sp} \leq 1)$ of the confirmed negative plates that did not show presumptive Salmonella colonies. The PPV and NPV was given as the proportion $(0 \leq \mathrm{PPV} \leq 1$ and $0 \leq \mathrm{NPV} \leq 1)$ of the positive Salmonella selective plates and negative selective plates that were confirmed as positive Salmonella selective plates and negative selective plates respectively.

\section{Results}

Out of 374 samples obtained from the poultry production setting in Calabar, 221 (59.1\%) were confirmed to be positive for Salmonella species as determined by the biochemical and serological confirmation characteristics (Table 1).

Table 2 shows the number of confirmed Salmonella positive platesfor the recovery of Salmonella species from MSRV and MKTTn selective enrichment media with XLD and BG selective plating media. Fewer confirmed Salmonella positive plates were obtained from XLD and BG plating media when MKTTn, rather than when MSRV enrichment medium was used. However, the difference was not statistically significant (P-value > 0.05). When XLD selective plating media was used, samples cultured in MSRV yielded 217 (98.2\%) confirmed Salmonella positive plates while samples cultured in MKTTn yielded 199 (90.0\%) confirmed Salmonella positive plates. When BG selective plating media was used, samples cultured in MSRV yielded 211 (95.5\%) confirmed Salmonella positive plates while samples cultured in MKTTn yielded 142 (64.3\%) confirmed Salmonella positive plates.

Table 3 shows the comparison of the test parameters (sensitivity, specificity, PPV and NPV) for the recovery of Salmonella species from samples following culture on selective plating media. The combination of MSRV/ XLD demonstrated the highest sensitivity (0.98), followed by MSRV/BG (0.95), MKTTn/XLD (0.90) and MKTTn/BG (0.71), while the highest specificity was observed in the combination of MSRV/BG (0.88) followed by MKTTn/BG (0.65), MSRV/XLD (0.50) and MKTTn/XLD (0.13). The PPV of presumptive Salmonella positive plates using MSRV/BG combinations was higher (0.92) (not significantly high) than any other combinations. The NPV of negative plates using MSRV/XLD (0.95) and MSRV/BG (0.93) combinations were higher (significantly high) than the combinations of MKTTn/XLD (0.48) and MKTTn/BG (0.63). The number of false positive XLD plates was higher than the number of false positive BG plates, particularly in the case of MKTTn/

Table 1. Detection of Salmonella from the primary production of poultry.

\begin{tabular}{cccc}
\hline Source of sample & No. of samples & No. positive & \% positive \\
\hline Poultry environment & 170 & 100 & 58.8 \\
Poultry bird & 136 & 75 & 55.1 \\
Poultry personnel & 68 & 56 & 67.6 \\
Total & 374 & 221 & 59.1 \\
\hline
\end{tabular}

Table 2. Positivity of selective plating media in combination with selective enrichment media.

\begin{tabular}{ccc}
\hline Selective plates & No. of presumptive positive plates & \\
\hline MSRV/XLD & 217 & Percentage of presumptive positive plates $^{-17}$ \\
MSRV/BG & 211 & 98.2 \\
MKTTn/XLD & 199 & 95.5 \\
MKTTn/BG & 142 & 90.0 \\
\hline
\end{tabular}

${ }^{a}=$ there was no statistical significant difference among the all the combinations of selective enrichments media with selective plates. MSRV/XLD = Xylose lysine deoxycholate agar in combination with Modified semisolid rappaport-vassiliadis enrichment medium; MSRV/BG = Brilliant green agar in combination with Modified semisolid rappaport-vassiliadis enrichment medium; MKTTn/XLD = Xylose lysine deoxycholate agar plate in combination with Muller-Kaufmann tertrathionate novobiocin; MKTTn/BG = Brilliant green agar plate in combination with Muller-Kaufmann tertrathionate novobiocin.

Percentage of presumptive plates $=\frac{\text { No. of presumptive positive plates }}{\text { No. of confirmed positive plates }} \times 100$ 
Table 3. Comparison of the selective plating media for the detection of Salmonella from enrichment media.

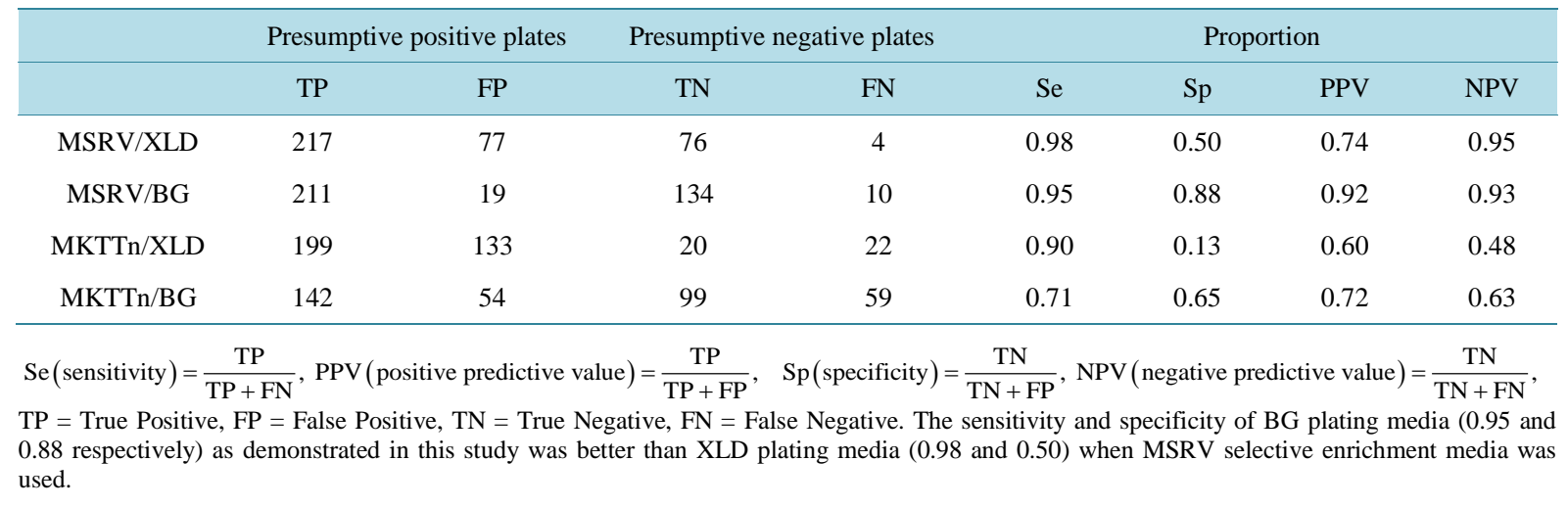

XLD combinations (133 false positive plates). The number of false negative BG plates was higher than the number of false negative XLD plates, particularly in the MKTTn/BG combinations (59 false negative plates).

\section{Discussion}

The recovery of Salmonella species from the primary production of poultry demonstrated a rate of $59.1 \%$. Several countries have recorded different rates of recovery of Salmonella species from poultry settings such as $70.5 \%$ in Brazil [23], 63.6\% in Ethiopia [24], 53.3\% in Vietnam [25], 47.7\% in Austria [26], 42.3\% in Korea [11], 17\% in USA [27], 10.1\% in Georgia [28] and 1\% in Jamaica [29]. Such varying rates of recovery of Salmonella can be attributed to differences in the geographical location, the standards of hygiene and sanitation practices observed in these regions, in addition to the methods used in the detection of Salmonella.

The rate of recovery of Salmonella species from the poultry production setting in this current study was higher compared to a previous study carried out in 2009 by Garba et al. in Yola, Nigeria, which recorded a 40.8\% rate of recovery of Salmonella species [30]. This result suggests an increasing trend of Salmonella in the poultry production system in Nigeria in addition to poor hygiene/sanitation practices in the primary poultry production settings in Nigeria. This has a great implication in that it poses a serious threat in terms of veterinary health which can lead to the loss of a greater majority of poultry birds and most importantly to the consumer population who may become infected. Moreover, this is in agreement with the report of Garba et al. (2010), who diagnosed more than $50 \%$ of the death of birds in Yola, Nigeria to be caused by Salmonella species [30], meanwhile several studies from different parts of the world have incriminated poultry and poultry products as the major source of salmonellosis in humans [6].

This current study was aimed at assessing the performance of XLD as preferential selective plating medium by comparing it with BG for the detection of salmonella in the primary production of poultry based on the ISO 6579:2002. The results of this study demonstrated fewer confirmed Salmonella positive plates from XLD and BG plating media when MKTTn was used, and more confirmed Salmonella positive when MSRV enrichment medium was used. This was in corroboration with those of several other studies which yielded more Salmonella positive plates when Rappaport-Vassiliadis rather than Tertrathionate selective enrichments were used [5] [11] [31]-[33]. Moreover, from the above observations, the combination of MSRV for selective enrichment and BG for selective plating media (MSRV/BG) may be the most appropriate in the detection and isolation of Salmonella from samples in the primary production of poultry.

Furthermore, in spite of the high sensitivity of XLD selective plating media over BG, the specificity of XLD was considerably low when compared to that of BG. This therefore affirms the superiority of MRSV/BG plating media over MSRV/XLD plating media for the detection of Salmonella species in poultry production systems. However, this is in disagreement with the traditional culture method for the isolation of Salmonella in feed stuff (ISO 6579, 2002), which uses XLD as the preferential selective plating media over the others (such as Brilliant Green agar, Salmonella Shigella agar, Hectoen Enteric agar etc.) for the isolation and detection of Salmonella. This contradiction may be as a result of the type of samples used in this study. The samples in this study included samples from the poultry environment (poultry feeds, poultry drinking water, poultry litter, poultry abattoir reins and dust from poultry house), from the poultry birds themselves (guts, cloacal swabs, poultry meat and 
eggs) and from the poultry personnel (poultry personnel stool and hand washings). Such a contradiction could have arisen as a result of the fact that different types of samples may be suitable for the detection of Salmonella using specific types of selective Plating media. Therefore, there is need for studies to be carried out to evaluate the performances of different selective plating media for the detection of Salmonella in different types of samples.

\section{Conclusions}

The results of this current study demonstrated that the type of selective enrichment media used (MSRV and MKTTn) had substantial effects on the sensitivity and specificity of the selective plating media (XLD and BG). MSRV selective enrichment medium was the most important, necessary for the investigation Salmonella in poultry production systems.

XLD plates demonstrated higher number of false positives than BG plates, while BG plates on the other hand demonstrated higher number of false negatives. In order to monitor food safety, it would be better to strike a bargain on a method that demonstrates fewer false positives than false negatives, of which BG selective plating media was the case with this current study. The combination of MSRV and BG revealed the highest sensitivity and specificity for the detection of Salmonella with fewer false positive plates. Therefore, based on the results of this study, the combination of MSRV/BG may render a better improvement for the detection of Salmonella, thereby minimising the cost of using two selective plating media in addition to the expensive and labour intensive biochemical and serological confirmations for the detection of Salmonella.

\section{Limitation of the Study}

MSRV is unable to detect non-motile Salmonella bacteria, which represent $<1.0 \%$ of the isolates from poultry environment.

\section{References}

[1] Akond, M.A., Shirin M., Alam, S., Hassan, S.M.R., Rahman, M. and Hoq, M. (2012) Frequency of Drug Resistant Salmonella spp. Isolated from Poultry Samples in Bangladesh. Stamford Journal of Microbiology, 2, 2074-5346.

[2] Yhiler, N.Y. and Bassey, B.E. (2015) Critical Segments in the Dissemination and Transmission of Salmonella Species from Poultry Production in Calabar, Nigeria. Science Journal of Public Health, 3, 168-174.

[3] Voetsch, A.C., Van Gilder, T.J., Angulo, F.J., Farley, M.M., Shallow, S., Marcus, R., Cieslak, P.R., Deneen, V.C. and Tauxe, R.V. (2004) FoodNet Estimate of the Burden of Illness Caused by Nontyphoidal Salmonella Infections in the United States. Clinical Infectious Disease, 38, S127-S134. http://dx.doi.org/10.1086/381578

[4] Velge, P.C. and Barrow, P. (2005) Emergence of Salmonella Epidemics: The Problem Related to Salmonella Enteric Serotype Enteriditis and Multiple Antibiotic Resistances in Other Major Serotypes. Veterinary Research, 36, 276-288. http://dx.doi.org/10.1051/vetres:2005005

[5] Schönenbrücher, V., Mallinson, E.T. and Bulte, M. (2008) A Comparison of Standard Cultural Methods for the Detection of Foodborne Salmonella Species Including Three New Chromogenic Plating Media. International Journal of Food Microbiology, 123, 61-66. http://dx.doi.org/10.1016/j.ijfoodmicro.2007.11.064

[6] Pui, C.F., Wong, W.C., Chai, L.C., Nillian, E., Ghazali, F.M., Cheah, Y.K., Nakaguchi, Y., Nishibuchi, M. and Radu, S. (2011) Simultaneous Detection of Salmonella spp., Salmonella Typhi and Salmonella Typhimurium in Sliced Fruits Using Multiplex PCR. Food Control, 22, 337-342. http://dx.doi.org/10.1016/j.foodcont.2010.05.021

[7] Velusamy, V., Arshak, K., Korostynska, O., Oliwa, K. and Adley, C. (2010) An Overview of Foodborne Pathogen Detection: In the Perspective of Biosensors. Biotechnology Advance, 28, 232-254. http://dx.doi.org/10.1016/j.biotechadv.2009.12.004

[8] Mooijman, K.A. (2004) The Use of Semi-Solid Media for the Detection of Salmonella spp. in Poultry Faeces and Other matrices. Working Document ISO/TC34 SC9 N681-Annex 1, 17.12.204.

[9] Andrews, W.H. and Hammack, T.S. (1998) Salmonella. Bacteriological Analytical Manual. 8th Edition, Revision A, Chapter 5. USFDA, White Oak, MD.

[10] ISO 6579:2002/Amd 1:2007, Annex D: Detection of Salmonella spp. in Animal Faeces and in Environmental Samples from the Primary Production Stage.

[11] Hyeon, J.Y., Chon, J.W., Hwang, I.G., Kwak, H.S., Kim, M.S., Kim, S.K., Choi, I.S., Song, C.S., Park, C. and Seo, K.H. (2011) Prevalence, Antibiotic Resistance, and Molecular Characterization of Salmonella serovars in Retail Meat 
Products. Journal of Food Protection, 74, 161-166. http://dx.doi.org/10.4315/0362-028X.JFP-10-327

[12] Hyeon, J.Y., Park, J.H., Chon, J.W., Wee, S.H., Moon, J.S., Kim, Y.J. and Seo, K.H. (2012) Evaluation of Selective Enrichment Broths and Chromogenic Media for Salmonella Detection in Highly Contaminated Chicken Carcasses. Poultry Science, 91, 1222-1226. http://dx.doi.org/10.3382/ps.2011-01936

[13] Van Schothorst, M. and Renaud, A.M. (1983) Dynamics of Salmonella Isolation with Modified Rappaport's Medium. Journal of Applied Bacteriology, 54, 209-215. http://dx.doi.org/10.1111/j.1365-2672.1983.tb02609.x

[14] Muller, L. (1923) Un nouveau milieu d'enrichissement pour la recherche du bacilletyphique et des paratyphiques. Comptes Rendus des Seances de la Societe de Biologie et des ses Filiales, 89, 434-447.

[15] Kauffmann, F. (1935) Weitere Erfahrungen mit dem kombinierten Anreicherungsverfahren für Salmonellabacillen. Journal of Food Hygiene, 117, 26-32. http://dx.doi.org/10.1007/BF02176950

[16] Jeffries, L. (1959) Novobiocin-Tetrathionate Broth: A Medium of Improved Selectivity for the Isolation of Salmonellae from Faeces. Journal of Clinical Pathology, 12, 568-571. http://dx.doi.org/10.1136/jcp.12.6.568

[17] ISO 6579 (2002) Microbiology-General Guidance on Methods for the Detection of Salmonella. (E) 4th Edition, International Organization for Standardization, Geneva.

[18] Kumar, R., Surendran, R.K. and Thampuran, N. (2010) Evaluation of Culture Media for Selective Enrichment and Isolation of Salmonella in Seafood. Journal of AOAC International, 93, 1468-1471.

[19] Manafi, M. (2000) New Developments in Chromogenic and Fluorogenic Culture Media. International Journal of Food Microbiology, 60, 205-218. http://dx.doi.org/10.1016/S0168-1605(00)00312-3

[20] Maciorowski, K.G., Herrera, P., Jones, F.T., Pillai, S.D. and Ricke, S.C. (2006) Cultural and Immunological Detection Methods for Salmonella spp. in Animal Feeds-A Review. Veterinary Research Community, 30, 127-137. http://dx.doi.org/10.1007/s11259-006-3221-8

[21] Akond, M.A., Alam, S., Hassan, S.M.R. and Shirin, M. (2009) Antibiotic Resistance of Escherichia coli Isolated from Poultry and Poultry Environment of Bangladesh. Internet Journal of Food Safety, 11, 19-23.

[22] Carrique-Mas, J.J., Barnes, S., McLaren, I. and Davies, R. (2009) Comparison of Three Plating Media for the Isolation of Salmonella from Poultry Environmental Samples in Great Britain Using ISO 6579:2002 (Annex D). Journal of Applied Microbiology, 107, 1976-1983. http://dx.doi.org/10.1111/j.1365-2672.2009.04386.x

[23] Kanashiro, A.M.I., Stoppa, G.F.Z., Cardoso, A.L.S.P., Tessari, E.N.C. and Castro, A.G.M. (2005) Serovars of Salmonella spp. Isolated from Broiler Chickens and Commercial Breeders in Diverse Regions in Brazil from July 1997 to December 2004. Revista Brasileira de Ciência Avícola, 7, 195-198. http://dx.doi.org/10.1590/S1516-635X2005000300010

[24] Molla, B.D., Alemayehu, L. and Salah, W. (2003) Sources and Distribution of Salmonella Serotypes Isolated from Food Animals, Slaughterhouse Poultry Handlers and Retail Meat Products in Ethiopia. Ethiopian Journal of Health Development, 17, 63-70. http://dx.doi.org/10.4314/ejhd.v17i1.9782

[25] Van, T.T., Moutafis, G., Istivan, T., Tran, L.T. and Coloe, P.J. (2007) Detection of Salmonella spp. in Retail Raw Food Samples from Vietnam and Characterization of Their Antibiotic Resistance. Applied Environmental Microbiology, 73, 6885-6890. http://dx.doi.org/10.1128/AEM.00972-07

[26] Pointon, A., Sexton, M., Dowsett, P., Saputra, T., Kiermeier, A., Lorimer, M., Holds, G., Arnold, G., Davos, D., Combs, B., Fabiansson, S., Raven, G., McKenzie, H., Chapman, A. and Sumner, J. (2008) A Baseline Survey of the Microbiological Quality of Chicken Portions and Carcasses at Retail in Two Australian States (2005 to 2006). Journal of Food Protection, 71, 1123-1134.

[27] Logue, C.M., Sherwood, J.S., Olah, P.A., Elijah, L.M. and Dockter, M.R. (2003) The Incidence of AntimicrobialResistant Salmonella spp. on Freshly Processed Poultry from US Midwestern Processing Plants. Journal of Applied Microbiology, 94, 16-24. http://dx.doi.org/10.1046/j.1365-2672.2003.01815.X

[28] Liljebjelke, K.A., Hofacre, C.L., Liu, T., White, D.G., Ayers, S.S.Y. and Maurer, J.J. (2005) Vertical and Horizontal Transmission of Salmonella within Integrated Broiler Production System. Foodborne Pathogens and Disease, 2, 90102. http://dx.doi.org/10.1089/fpd.2005.2.90

[29] Curtello, S., Justiz, A.A., Vaillant, H., Asemota, P.E.A. and Monica, P.S. (2013) Prevalence of Salmonella Organisms in Poultry and Poultry Environments in Jamaica. British Microbiology Research Journal, 3, 461-469. http://dx.doi.org/10.9734/BMRJ/2013/4255

[30] Garba, A., Bolajoko, B.M., Barde, A., Ahmed, I., Sa’adatu, I., Agang, A.S., Abdullahi, H.A., Bakari, U.I.J., Uraki, A.T., Abdurrahman, A. and Goji, J.N. (2010) The Threat of Salmonellasis to Commercial Poultry Production in Adamawa State, Nigeria. Sokoto Journal of Veterinary Sciences, 8, 50-52.

[31] June, G.A., Sherrod, P.S., Hammack, T.S., Amaguana, R.M. and Andrews, W.H. (1996) Relative Effectiveness of Selenite Cysteine Broth, Tetrathionate Broth, and Rappaport-Vassiliadis Medium for Recovery of Salmonella spp. from 
Raw Flesh, Highly Contaminated Foods, and Poultry Feed: Collaborative Study. Journal of AOAC International, 79, 1307-1323.

[32] Rall, V.L., Rall, M.R., Aragon, L.C. and Silva, M.G. (2005) Evaluation of Three Enrichment Broths and Five Plating Media for Salmonella Detection in Poultry. Brazilian Journal of Microbiology, 36, 147-150.

http://dx.doi.org/10.1590/S1517-83822005000200009

[33] Pal, A. and Marshall, D.L. (2009) Comparison of Culture Media for Enrichment and Isolation of Salmonella spp. from Frozen Channel Catfish and Vietnamese Basa Fillets. Food Microbiology, 26, 317-319. http://dx.doi.org/10.1016/j.fm.2008.12.003 